\title{
Application of Traditional Chinese Medicine in Treatment of Atrial Fibrillation
}

\author{
Yan Dong, ${ }^{1}$ Jiangquan Liao, ${ }^{1,2}$ Kuiwu Yao, ${ }^{1}$ Wenrui Jiang, ${ }^{1}$ and Jie Wang1 \\ ${ }^{1}$ Department of Cardiology, Guang'anmen Hospital, China Academy of Chinese Medical Sciences, Beijing, China \\ ${ }^{2}$ Graduate School, Beijing University of Chinese Medicine, Beijing, China
}

Correspondence should be addressed to Jie Wang; wangjie0103@126.com

Received 13 September 2016; Revised 20 December 2016; Accepted 9 January 2017; Published 24 January 2017

Academic Editor: I-Min Liu

Copyright (C) 2017 Yan Dong et al. This is an open access article distributed under the Creative Commons Attribution License, which permits unrestricted use, distribution, and reproduction in any medium, provided the original work is properly cited.

\begin{abstract}
Atrial fibrillation (AF) is the most common cardiac arrhythmia, which is related to many cardiac and cerebral vascular diseases, especially stroke. It can therefore increase cardiovascular mortality and all-cause death. The current treatments of AF remain to be western drugs and radiofrequency ablation which are limited by the tolerance of patients, adverse side effects, and high recurrence rate, especially for the elderly. On the contrary, traditional Chinese medicine (TCM) with long history of use involves various treatment methods, including Chinese herbal medicines (CHMs) or bioactive ingredients, Chinese patent medicines, acupuncture, Qigong, and Tai Chi Chuan. With more and more researches reported, the active roles of TCM in AF management have been discovered. Then it is likely that TCM would be effective preventive means and valuable additional remedy for AF. The potential mechanisms further found by numerous experimental studies showed the distinct characteristics of TCM. Some CHMs or bioactive ingredients are atrial-selective, while others are multichannel and multifunctional. Therefore, in this review we summarized the treatment strategies reported in TCM, with the purpose of providing novel ideas and directions for AF management.
\end{abstract}

\section{Introduction}

Atrial fibrillation (AF) is the most common cardiac arrhythmia which will continue to grow rapidly. The estimated lifetime risk of developing $\mathrm{AF}$ is $25 \%$ in people over 40 years [1]. Another investigation [2] showed that the elderly had higher prevalence than the young. Current four principles of AF treatment include rate control, rhythm control, antithrombotic therapy, and dealing with the primary disease (or risk factors). So far western medicines remain the major treatment strategy of rate and rhythm control. Their positive actions are definite in emergency situations but still limited by recurrence of AF, visceral injuries, and inevitable adverse effects, such as severe ventricular arrhythmia [3], especially for long-time use. Another effective option is catheter ablation, which however has high recurrence and patients often have to receive once more operations. So this is one bottleneck met in the treatment of AF. The other one neck of bottle occurs in antithrombotic therapy which contains anticoagulation, antiplatelet aggregation, and fibrinolytic in general. All of them have high risk of bleeding, especially for the old persons. Even if antithrombotic therapy is the key of AF treatment, many patients have not received enough treatment or have been in poor INR control $[4,5]$, owing to the factors of both doctors and the patients [6]. Thereby in spite of some progress made in AF management, there still exists many critical problems.

On the contrary, traditional Chinese medicine (TCM), as complementary and alternative treatment, therefore becomes a viable option for these AF patients. It is reported that some single Chinese herbal medicines (CHMs) or bioactive ingredients, traditional Chinese patent medicines, and nondrug methods including acupuncture, Qigong, and Tai Chi Chuan can play active roles in the four principles of AF treatment, respectively, with low adverse reaction. What is more, they can lower the degree of discomforts like palpitation, chest discomfort, and dyspnea and then increase the tolerance of patients to disease, thus obviously improving their life quality. Further, multichannels are reportedly involved in the action mechanisms, including regulation of ion channels $[7,8]$, inhibition of inflammatory factors [9], activity of antioxidant [10, 11], and effect of antiplatelet aggregation [12] 
Additionally, some CHMs or bioactive ingredients selectively work on the atrial myocytes, thus avoiding the property of proarrhythmia. In this review, we summarized these distinct types of TCMs with the purpose of demonstrating the alternative treatment of AF in perspective of TCM.

\section{Single CHMs or Bioactive Ingredients}

CHMs have long history of use basis with the theory of TCM in China. A former research showed around 13,000 herbs were estimated to be usually used, and more than 100,000 medicinal recipes had been recorded in China [13]. These data are bound to be updated since the wider acceptance and usage now than before. The effectiveness and safety of these herbs are confirmed by more and more researches, and potential mechanisms are gradually being discovered. AF, as a common refractory disease, has attracted people over the world to study therapeutic Chinese herbs targeting itself, which in return highlights the advantages of CHMs.

2.1. Berberis vulgaris L. Berberis vulgaris L. (1753) is the most well-known species of Berberis that belongs to family Berberidaceae and evergreen shrubs throughout the temperate and subtropical regions of the world (apart from Australia). Its berries were used for culinary purposes in ancient Europe and Iran, and the flowers had the function of treating musculoskeletal pain in the theory of TCM for many centuries while current researches have focused on the extract from barberry root, berberine, which belongs to isoquinoline alkaloid. Berberine is considered to be a potential agent for AF because of its effects on primary disease, rate control, and rhythm control. It is reported to have these medicinal properties of vasodilation, positive ionotropic, and negative chronotropic actions [7], by which some primary diseases of AF like coronary heart disease and heart failure can be relieved. Further, it can suppress acetylcholine-induced AF in the rabbit through increasing effective refractory period (ERP) and prolonging the action potential duration (APD) of atrial myocytes [14]. This function was demonstrated to be in a dose-dependent manner [7] and in the biochemical mechanism of inhibiting the integral of transient outward potassium current (Ito) [7]. Moreover, berberine is a typical multichannel ion blocker. Previous studies have showed that it can inhibit KATP [15], IKV [16], IKCa [16], IK1, and IK [17]. Regrettably, though berberine shows promising function on AF (Table 1), the advantages have not been systematically studied in human clinical trials. And we should use it with caution, for it is demonstrated to be a potent inhibitor of CYP3A4 enzyme which involves in the metabolism of many medications [18].

\subsection{Saussurea involucrata. Saussurea involucrata (Kar. \&} Kir.) Sch. Bip. (1846), named snow lotus in central Asia, is a genus of flowering plants in the thistle tribe within the family Asteraceae. It is perennial herbaceous plant and precious medicinal material, with the treatment of musculoskeletal pain and tonifying kidney effect in ancient China. Saussurea flowers and stems are therefore widely used in rheumatic disease. Recent studies further illustrated that Saussurea and the sesquiterpene lactone fraction of Saussurea lappa roots had anti-inflammatory and analgesic effects [19-21]. In addition, the flavone compound of Saussurea, acacetin, was showed to prolong APD and ERP without prolonging the corrected QT interval in atrial myocytes [8], thus suppressing $\mathrm{AF}$ in dogs. The potential mechanism was the inhibition of acetylcholine-activated potassium current (Kach), ultrarapid delayed rectifier (IKur), and transient outward (Ito) potassium current as an atrial-selective agent [8]. Compared with the TCM theory, acacetin is a new discovery which is an effective and promising choice for AF (Table 1), but limited to animal experiments. Moreover, researchers failed to discover the relationship between anti-inflammatory and restraint myocardial remodeling, which may provide new direction for preventing the occurrence and development of AF. Therefore, further studies are needed to excavate the potential mechanism and relevant clinical investigations are urgently required.

2.3. Crataegus rhipidophylla Gand. Crataegus rhipidophylla Gand. (1872) is also called hawthorn that belongs to a large genus of shrubs and trees in the family Rosaceae. It is believed to promote blood to run around the whole body in the TCM theory. Based on this theory, a research discovered that two proanthocyanidins of its flower heads, catechin and epicatechin, inhibited the biosynthesis of thromboxane A2, leading to antiplatelet effect, but may increase the risk of bleeding [12]. Currently, hawthorn is widely used in cardiovascular diseases, especially arrhythmia [22], congestive heart failure [23-26], and hypertension [27]. Studies demonstrated that the biochemical mechanism of antiventricular arrhythmia is prolonging the APD through blocking the delayed (IK) and inward (IK1) rectifier potassium currents [22]. However, Long et al. [28] preferred that hawthorn was consistent with the effects profile of phosphodiesterase-3 (PDE3) inhibitors, which was different from Muller's opinion [22]. Hawthorn extract LI 132 (Faros ${ }^{\circledR}$ 300, CRA) was further found to increase cardiac contractility with prolonging the ERP, thus avoiding arrhythmogenic potential [26]. In addition, the other extract, WS 1442, would improve exercise capacity and then decrease mortality in heart failure patients [24, 25]. Furthermore, it was indicated that the epicatechin and hyperoside in the hawthorn fruit tincture [29] and the fluid extract of hawthorn had antioxidant activity [30]. From the above results, we find hawthorn extracts have the characteristics of antiplatelet aggregation, rhythm control, antioxidant activity, and management of the primary disease like heart failure, which makes it possible agent for AF treatment (Table 1). Nevertheless, the identity of the cardioactive constituent is still uncertain [31]. Therefore, corresponding experimental and clinical researches targeting hawthorn on AF are needed and deserved.

2.4. Corydalis turtschaninovii Besser. Corydalis turtschaninovii Besser (1834) is a medicinally important species of Corydalis, which comes from the family Papaveraceae. Its 


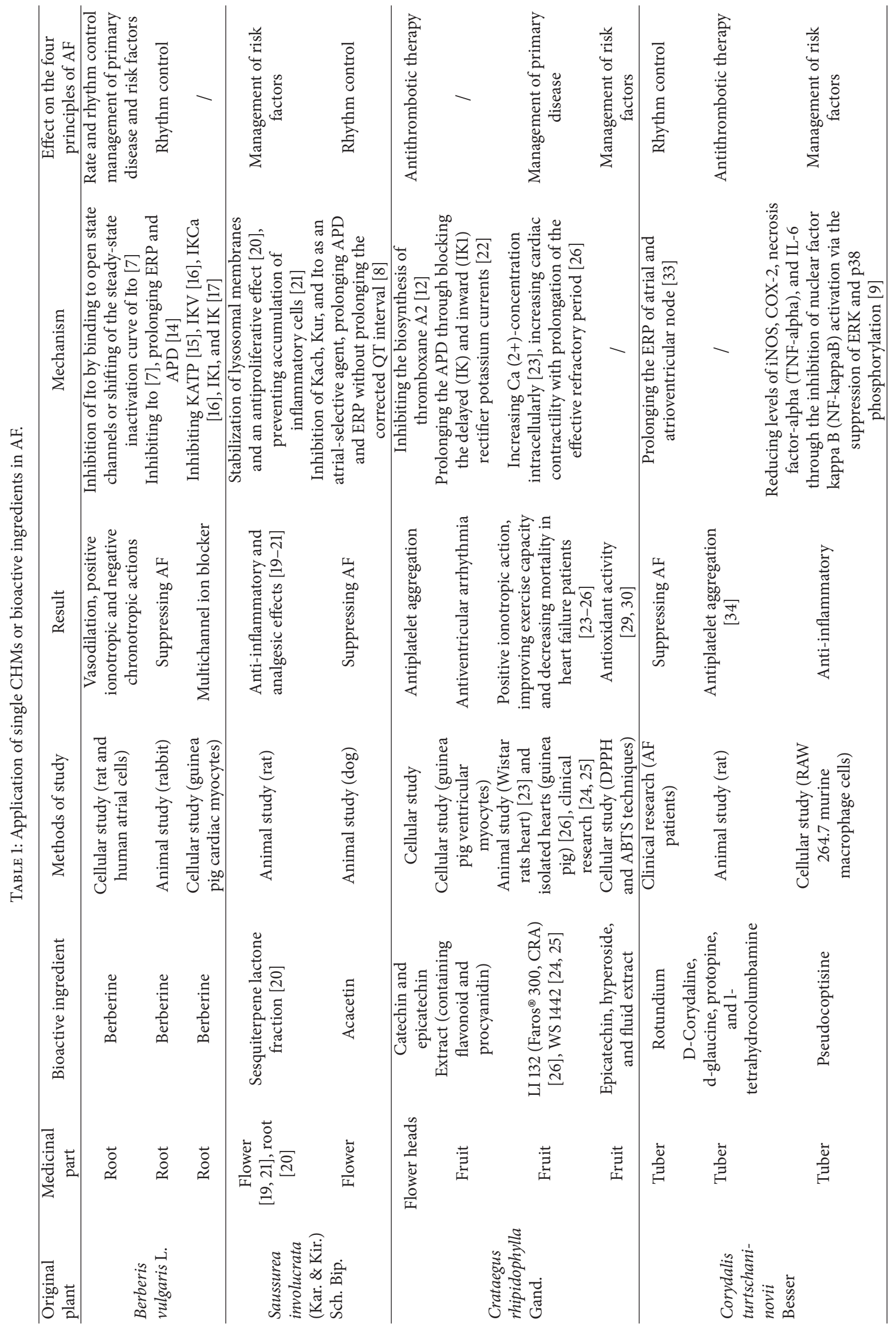




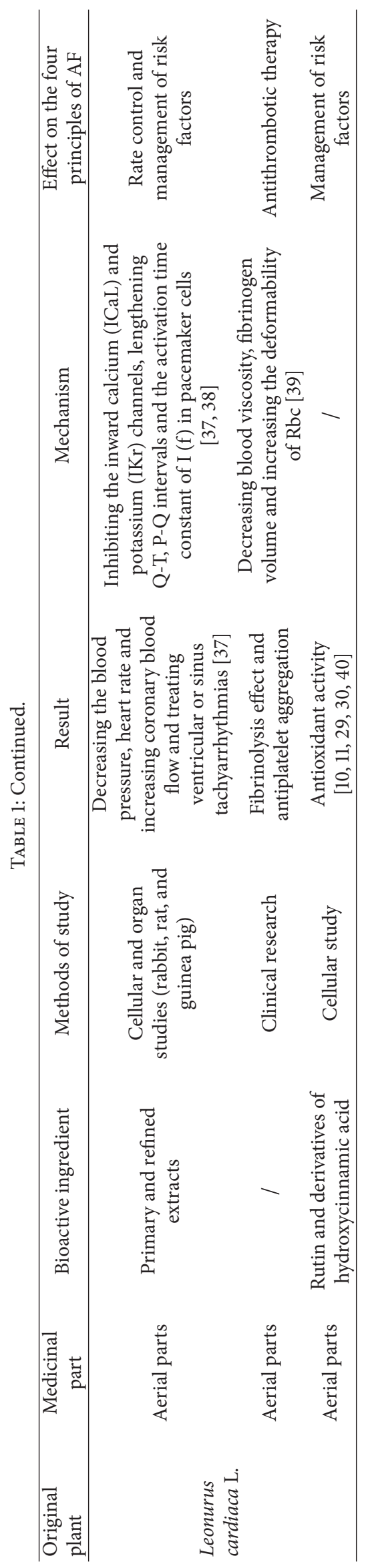


dried tubers, also known as Yanhusuo in China, can invigorate the circulation of blood and relieve pains, thus having been widely used in the treatment of cardiovascular diseases including arrhythmia [32]. Previous pharmacological studies reported that rotundium, an alkaloid of Corydalis turtschaninovii was antiarrhythmic by blocking the calcium channel in some animal experiments. And a later clinical research demonstrated that rotundium was an effective and safe medicine to treat AF, especially paroxysmal AF. The corresponding mechanism may be prolonging the ERP of atrial and atrioventricular node [33]. Except rotundium, there are four other alkaloids, d-corydaline, d-glaucine, protopine, and l-tetrahydrocolumbamine, isolated from the methanol extract of Chinese Corydalis tuber (CMe), which showed inhibitory action on blood platelet aggregation [34]. And the CMe was found to inhibit the decrease of blood platelets in disseminated intravascular coagulation (DIC) and inhibit pulmonary thromboembolism [34]. In addition, pseudocoptisine, a quaternary alkaloid with a benzylisoquinoline skeleton, was also extracted from the Corydalis turtschaninovii tubers and showed to have anti-inflammatory property. The potential molecular mechanism was reducing levels of the proinflammatory mediators, such as iNOS, COX2, necrosis factor-alpha (TNF-alpha), and IL-6 through the inhibition of nuclear factor kappa B (NF-kappaB) activation via the suppression of ERK and p38 phosphorylation in RAW 264.7 cells [9]. Taken together, we can find that Corydalis turtschaninovii or its methanol extract has rhythm control or antiplatelet aggregation function. What is more, it can inhibit the inflammatory response that involves myocardial remodeling and thrombosis $[35,36]$. Therefore, Corydalis turtschaninovii is a multifunctional agent (Table 1), which is worthy of in-depth researches targeting AF.

2.5. Leonurus cardiaca L. Leonurus cardiaca L. (1753), known as motherwort as well, is a herbaceous perennial plant in the mint family, Lamiaceae. It can be found worldwide, spreading largely due to its use as a herbal remedy. Motherwort with long history of use as a traditional herb in Asia is often used for uterine infection or other gynecological diseases in TCM, while the aqueous extracts from the aerial parts of Leonurus cardiaca have been used as a remedy against tachyarrhythmia and other cardiac disorders in Europe [37]. A research demonstrated that the primary and refined extracts of Leonurus cardiaca L. can inhibit the inward calcium (ICaL) and potassium (IKr) channels and lengthen Q-T, P-Q intervals and the activation time constant of I (f) in pacemaker cells, which lead to prolongation of cardiac cycle and activation recovery interval and APD in sinoatrial node cells and ventricular myocytes [37, 38]. Thus they decrease the blood pressure and heart rate and increase coronary blood flow and treat ventricular or sinus tachyarrhythmias [37]. In addition, motherwort also decreased blood viscosity and fibrinogen volume and increased the deformability of Rbc and antiplatelet aggregation effect [39]. Moreover, extracts from motherwort including polyphenolic compounds, mainly flavonoids (rutin) and derivatives of hydroxycinnamic acid, demonstrated antioxidant activity in several in vitro studies $[10,11,29,30,40]$. With the function of rate control, fibrinolysis, and antiplatelet aggregation effect and antioxidant activity which may be useful for addressing the primary disease (Table 1), motherwort or its extracts are promising and deserve further research in AF patients.

\section{Traditional Chinese Patent Medicines}

Traditional Chinese patent medicines are mainly comprised of Chinese herbal medicines or their extracts. With large numbers of clinical and experimental researches, many Chinese classical formulas are currently made into patent medicines for their efficacy, safety, and convenience. Therefore, more and more traditional Chinese patent medicines for specific diseases including AF are produced and used clinically.

3.1. Wenxin Granule (WXG). Wenxin granule (WXG) is a Chinese medication, which contains Codonopsis pilosula Nannf., Polygonatum sibiricum Red., Panax notoginseng, Nardostachys chinensis Batal., and amber. WXG reportedly benefited patients with atrial arrhythmias and heart failure [41]. It is indicated to shorten conversion time, decrease the required dosage of amiodarone, and avoid the adverse reaction of longterm use of amiodarone, when combined with amiodarone [42]. A recent meta-analysis concluded that WXG alone or in combination with other antiarrhythmics decreased the recurrence of paroxysmal AF, with very few adverse effects [43]. The potential mechanism was demonstrated to be the inhibition of sodium channels (INa) selectivity in atrium, owing to more negative steady-state inactivation, less negative resting membrane potential, and shorter diastolic intervals in atrial versus ventricular cells at rapid activation rates [41, 44]. In addition, WXG produced prolongation of the ERP selectively in the atrial cardiomyocytes, without APD prolongation, thus lengthening the $\mathrm{P}$-wave duration and preventing persistent AF [41]. This is a novel property, especially APD shortening and postrepolarization refractoriness in an atrial-selective manner, which is controversial to the well-known paradigm that efficacious atrial specific antiarrhythmic drugs have to significantly prolong APD and/or wave length [45]. What is more, another thought-provoking aspect was put forward by Kalifa and Avula [46]. They pointed out the fact that WXG significantly shortened APD90 in a manner that is not merely compatible with the late INa blockade, which usually produces only moderate APD shortening. So other ion currents besides INa are assumed in this performance. The ultrarapid delayed rectified potassium current (IKur) also presents in atria but not ventricles in human heart $[47,48]$. So whether IKur is also participating in the action of WXG on AF deserves further discussion and research. Recently attentions have been already paid to developing selective inhibitors of the human atrial IKur or hKv1.5 channels [49], which may be a good news for AF patients. Additionally, WXG was found to lower AF inducibility after the ganglionic plexi (GP) ablation, without increasing the levels of atrial natriuretic peptide (ANP), tumour necrosis factor-alpha (TNF- $\alpha$ ), interleukin(IL-) 6, and expression of connexin 43 in atrial tissues, 
thus suppressing atrial substrate remodeling induced by GP ablation [50]. Although the current randomized controlled trials of WXG in treating AF (Table 2) are evaluated as low $[43,51]$, it surely shows certain treatment in different investigations and attracts people's attention to TCM.

\subsection{ShenSongYangXin Capsule (SSYXC). ShenSongYangXin} capsule (SSYXC) is another Chinese medication which is made up of Panax ginseng, Ophiopogon japonicus, Cornus officinalis Sieb., Salvia miltiorrhiza Bge., Ziziphus jujuba Mill., Taxillus chinensis Danser, Paeonia lactiflora Pall., ground beetle, Nardostachys jatamansi, Coptis chinensis Franch., Schisandra sphenanthera Rehd., and fossilia ossia mastodi. SSYXC has been used for the treatment of tachyarrhythmias [52]. A randomized, double-blind, and controlled multicenter research has been conducted to find that SSYXC has efficacy in the treatment of paroxysmal AF, which is similar to propafenone [53]. It prolonged the APD significantly, with the potential mechanism of depressing the L-type calcium channel current (ICa-L), transient outward potassium current (Ito), and inward rectifier potassium current (IK1) in a concentration-dependent manner [54]. And the adverse reaction rate of this medication is indicated to be low [53]. Additionally, SSYXC is used for premature ventricular contractions and ventricular arrhythmias as well $[55,56]$, possibly by blocking multiple ion channels, namely, INa, ICaL, Ito, and IK1 $[57,58]$. That is to say, it is a broad-spectrum antiarrhythmic drug, rather than the atrium-selective one. A meta-analysis [59] involving 22 trials and 2,347 paroxysmal AF (PAF) patients showed that although SSYXC appeared to improve P-wave dispersion (Pwd) and the frequency of PAF, the results included were inconsistent. Therefore, in order to further confirm the role of SSYXC (Table 2), more rigorous researches are needed to be done.

3.3. Shenmai Injection. Shenmai injection is derived from a Chinese classical formula which is an important component of TCM and has effective function of improving palpitation symptom. The injection is composed of ginseng saponins, radix ophiopogonis saponins, radix ophiopogonis flavonoids and traces of ginseng polysaccharides, and radix ophiopogonis polysaccharide as well. A current research showed that it could prolong ERP and convert AF into sinus rhythm, thus having synergistic effects with amiodarone [60] (Table 2). However, more reportedly studies are lacked to evaluate the function of shenmai injection on AF, and the potential mechanism is still unknown. Therefore, large numbers of experimental and clinical researches are urgently needed.

\section{Nondrug Methods}

TCM involves other therapeutic methods besides drugs, including acupuncture and Qigong that are illustrated to be effective in AF. Elderly people are the crowds in high risk of developing AF, with low level of liver and kidney condition; thus they easily get into drug intoxication. Therefore, nondrug methods are viable alternative for old patients and those with low tolerance for drugs.
4.1. Acupuncture. Acupuncture is an important and indispensable part of TCM. According to the traditional theory, many meridians and acupoints have antiarrhythmic effect, especially the Meridian of Minister of Heart that is associated with heart rate and blood flow. Neiguan spot is a spot in the area of this meridian, which locates in the middle of the forearm between the tendons [61]. It is an acupoint with high frequency of use and usually taken as the main spot for treating AF. Based on puncturing of the Neiguan, Shenmen, and Danzhong spots, a research probed the feasibility of acupuncture in conversion of paroxysmal AF and atrial flutter. The results showed that the rate of cardioversion was higher [62] and the duration to cardiovert $[62,63]$ was also shorter in patients treated with acupuncture than amiodarone. As to persistent AF, other studies have suggested that acupuncturing Neiguan, Shenmen, and Xinshu spots and so forth helped to decrease recurrences of persistent AF after cardioversion [63, 64], whose action was similar to amiodarone. In addition, the combination of acupuncture and Chinese patent medicine is perhaps a better choice for AF. A study indicated that, combined to simple Wenxin granule therapy in the treatment of paroxysmal AF, acupuncture combined with Wenxin granule had a better effect [65]. Therefore, acupuncture could be an effective nondrug and rhythm control tool in the management of these patients (Table 3). However, recent studies evaluating the effect of acupuncture are limited by small sample sizes and need to be validated in a larger population. And researches evaluating the mechanism of action have not yielded consistent results [66]; thus more experiments are ought to be conducted.

4.2. Qigong. Qigong is a special and unique part of TCM. Through physical exercises, it regulates the Qi of human body, which is a kind of material with important function, thus achieving health protection and treatment of diseases. Pippa et al. [67] confirmed that Qigong training was well tolerated and, compared with baseline, trained AF patients had better functional capacity and physical rehabilitation. In addition, Baduanjin, as one mind-body exercise of Qigong, could promote multisystem or organ functions (e.g., digestive and circulatory systems), increase immunity, make bodies relax, and improve mood and confidence of elderly populations [68]. Investigations further showed that Baduanjin was an effective therapy for hypertension [69] and dyslipidemia [70] and could reverse adverse left ventricular remodeling in post-myocardial infarction patients [71]. Additionally, it had beneficial effects on increasing antioxidant enzymes and reducing oxidative stress in middle-aged women by increasing superoxide dismutase and reducing malondialdehyde level [72]. Although Baduanjin was shown to be primary prevention of stroke in community old population with high risk factors [73], more rigorously designed RCTs about Baduanjin or Qigong and AF are still warranted. Therefore, Qigong is a promising and noninvasive method for management of primary diseases and risk factors for AF patients (Table 3), which need further mechanism researches. 


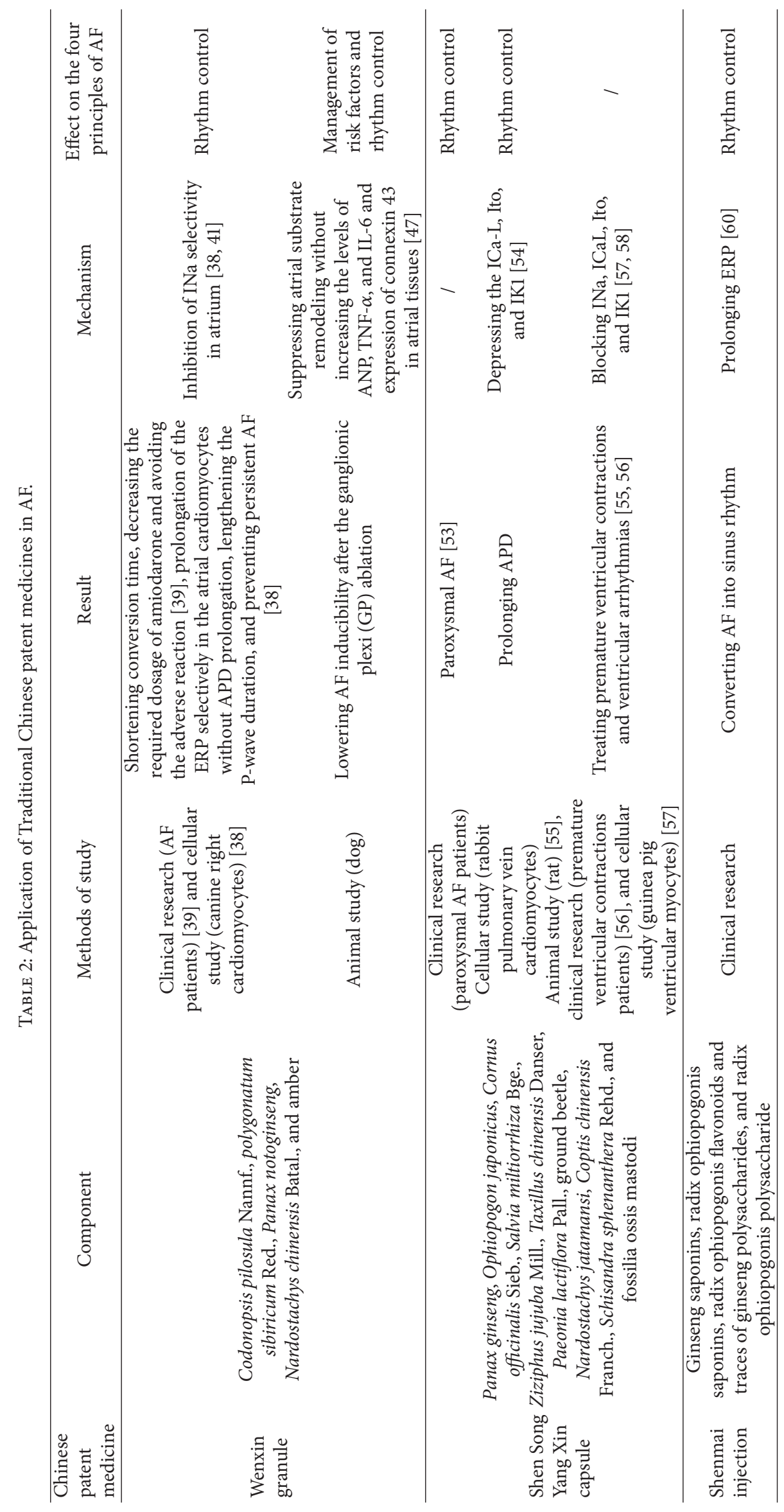


TABLE 3: Application of nondrug methods in AF.

\begin{tabular}{|c|c|c|c|}
\hline Name & Methods of study & Result & $\begin{array}{c}\text { Effect on the four principles } \\
\text { of AF }\end{array}$ \\
\hline \multirow[t]{2}{*}{ Acupuncture } & $\begin{array}{c}\text { Clinical research (paroxysmal AF and atrial } \\
\text { flutter patients) }\end{array}$ & $\begin{array}{l}\text { Increasing the rate of cardioversion [62], } \\
\text { lowering the duration of conversion time } \\
\qquad[62,63]\end{array}$ & Rhythm control \\
\hline & $\begin{array}{l}\text { Clinical research (paroxysmal and persistent } \\
\text { AF patients) }\end{array}$ & $\begin{array}{c}\text { Decreasing recurrences of persistent AF } \\
\text { after cardioversion }[63,64]\end{array}$ & Rhythm control \\
\hline \multirow{6}{*}{$\begin{array}{l}\text { Qigong } \\
\text { (including } \\
\text { Baduanjin) }\end{array}$} & Clinical research & Improving physical rehabilitation of AF [67] & 1 \\
\hline & Clinical research & $\begin{array}{c}\text { Reversing adverse left ventricular } \\
\text { remodeling }[71]\end{array}$ & Management of risk factors \\
\hline & Clinical research & $\begin{array}{l}\text { Promoting multisystem or organ functions, } \\
\text { increasing immunity, making bodies relax, } \\
\text { and improving mood and confidence of } \\
\text { elderly populations [68] }\end{array}$ & Management risk factors \\
\hline & Clinical research & Lowering blood pressure [69] & $\begin{array}{l}\text { Management of primary } \\
\text { disease }\end{array}$ \\
\hline & Clinical research & Modulating the blood lipid metabolism [70] & $\begin{array}{l}\text { Management of primary } \\
\text { disease }\end{array}$ \\
\hline & Clinical research & $\begin{array}{l}\text { Antioxidant activity and reducing oxidative } \\
\text { stress [72] }\end{array}$ & Management of risk factors \\
\hline \multirow{4}{*}{ Tai Chi Chuan } & Clinical research & $\begin{array}{l}\text { Improvement of cardiorespiratory function } \\
\text { [74] }\end{array}$ & Management of risk factors \\
\hline & Clinical research & Reducing CVD risk factors [75] & Management of risk factors \\
\hline & A meta-analysis & Lowering blood triglyceride level [76] & $\begin{array}{l}\text { Management of primary } \\
\text { disease }\end{array}$ \\
\hline & A literature search & Improvement in mental health [77] & Management of risk factors \\
\hline
\end{tabular}

4.3. Tai Chi Chuan (TCC). Tai Chi Chuan (TCC) is also known as shadow boxing. It is another way of physical exercise that is a combination of martial art and the regulation of Qi in the theory of TCM. It integrates the breath, mind, and physical activity, thus making exercisers achieve greater awareness and a sense of inner peace. TCC has been popularly accepted and practised as a health care approach and an alternative method for the reduction of symptoms and risk factors in cardiovascular diseases (Table 3). A recent research further indicated its efficacy in the improvement of cardiorespiratory function for old adults [74]. And a metaanalysis demonstrated that TCC lowered blood triglyceride level with a trend to decrease blood total cholesterol level [76]. What is more, as an aerobic exercise of moderate intensity, TCC could maintain better health and improve quality of life, thus reducing CVD risk factors [75]. In addition, TCC intervention could produce positive effects on patients with mental problems [77]. Therefore, TCC would be a good option for cardiovascular patients and serves as an adjunctive exercise modality to rehabilitation programs for patients with $\mathrm{CHD}$ or $\mathrm{CHF}$ [78]. However, we failed to find direct researches concerning TCC and AF, which has a scientific priority for future investigation.

\section{Discussion}

TCMs showed in the above researches indicate that they can work on more than one principle of AF management simultaneously through multichannels. By means of regulating ion channels $[7,8,22,33,37,38,41,54]$, they act positive roles in rhythm or rate control. They also give a hand to the risk factors by vasodilation [7], anti-inflammatory effect $[9,19]$, antioxidant activity $[10,30,72]$, and even resistance of myocardium remodeling $[50,71]$ and benefit some primary diseases such as heart failure [7, 26, 41], hypertension $[27,69]$, and dyslipidemia [70, 76]. In addition, antiplatelet aggregation [12, 34, 39] and fibrinolysis effect [39] are showed in some CHMs or bioactive ingredients. Therefore, TCMs have four significant characters that can be put forward here. To begin with, some medicines including Saussurea involucrata, Corydalis turtschaninovii Besser, and Wenxin granule can selectively work on the atrial myocytes with no effect on ventricular electrical parameters. It is just the one that the current strategy for suppressing AF is developing $[48,79]$ and the one that existing antiarrhythmic drugs lack. Next, the multichannels and multifunctions increase the therapeutic management of AF but reduce the side effects and the amount of drugs that only target one principle. Moreover, since exercise capacity [23], physical rehabilitation [67], and some mental problems [77] can be improved by TCMs, especially the nondrug methods, patients' life quality can be markedly enhanced. Finally, as AF is age-related and the old person has low tolerance for drugs because of declined function of liver and kidney, TCMs which have low adverse reaction $[43,53]$ will be a better choice for them. Nevertheless, two problems still exist in the current results, 
namely, the relatively insufficient and poor clinical studies and the limitation in anticoagulation.

In conclusion, despite suffering from lack of adequate mechanistic and clinical studies, TCM research in this area is exciting and may lead to the development of new drugs just like the discovery of amiodarone which is extracted from a plant named Ammi visnaga.

\section{Competing Interests}

The authors declare that they have no conflict of interests.

\section{Acknowledgments}

This work was supported by the special project of Beijing science and technology commission (no. Z161100001816045).

\section{References}

[1] P. Kirchhof, A. Auricchio, J. Bax et al., "Outcome parameters for trials in atrial fibrillation: executive summary-recommendations from a consensus conference organized by the German Atrial Fibrillation Competence NETwork (AFNET) and the European Heart Rhythm Association (EHRA)," European Heart Journal, vol. 28, no. 22, pp. 2803-2817, 2007.

[2] A. S. Go, E. M. Hylek, K. A. Phillips et al., "Prevalence of diagnosed atrial fibrillation in adults: National implications for rhythm management and stroke prevention: the anticoagulation and risk factors in atrial fibrillation (ATRIA) study," Journal of the American Medical Association, vol. 285, no. 18, pp. 2370-2375, 2001.

[3] D. M. Roden and M. E. Anderson, "Proarrhythmia," in Basis and Treatment of Cardiac Arrhythmias, vol. 171 of Handbook of Experimental Pharmacology, pp. 73-97, Springer, Berlin, Germany, 2006.

[4] E. W. Chan, W. C. Y. Lau, C. W. Siu et al., "Effect of suboptimal anticoagulation treatment with antiplatelet therapy and warfarin on clinical outcomes in patients with nonvalvular atrial fibrillation: a population-wide cohort study," Heart Rhythm, vol. 13, no. 8, pp. 1581-1588, 2016.

[5] C.-W. Siu, G. Y. H. Lip, K.-F. Lam, and H.-F. Tse, "Risk of stroke and intracranial hemorrhage in 9727 Chinese with atrial fibrillation in Hong Kong," Heart Rhythm, vol. 11, no. 8, pp. 14011408, 2014.

[6] V. W. Y. Lee, C. S. Tam, B. P. Yan, C. Man Yu, and Y. Yin Lam, "Barriers to warfarin use for stroke prevention in patients with atrial fibrillation in Hong Kong," Clinical Cardiology, vol. 36, no. 3, pp. 166-171, 2013.

[7] J.-F. Chi, S.-H. Chu, C.-S. Lee, N.-K. Chou, and M.-J. Su, "Mechanical and electrophysiological effects of 8-oxoberberine (JKL1073A) on atrial tissue," British Journal of Pharmacology, vol. 118, no. 3, pp. 503-512, 1996.

[8] G.-R. Li, H.-B. Wang, G.-W. Qin et al., "Acacetin, a natural flavone, selectively inhibits human atrial repolarization potassium currents and prevents atrial fibrillation in dogs," Circulation, vol. 117, no. 19, pp. 2449-2457, 2008.

[9] K.-J. Yun, J.-S. Shin, J.-H. Choi, N.-I. Back, H.-G. Chung, and K.-T. Lee, "Quaternary alkaloid, pseudocoptisine isolated from tubers of Corydalis turtschaninovi inhibits LPS-induced nitric oxide, PGE2, and pro-inflammatory cytokines production via the down-regulation of NF- $\kappa$ B in RAW 264.7 murine macrophage cells," International Immunopharmacology, vol. 9, no. 11, pp. 1323-1331, 2009.

[10] A. Matkowski and M. Piotrowska, "Antioxidant and free radical scavenging activities of some medicinal plants from the Lamiaceae," Fitoterapia, vol. 77, no. 5, pp. 346-353, 2006.

[11] A. Matkowski, P. Tasarz, and E. Szypuła, "Antioxidant activity of herb extracts from five medicinal plants from Lamiaceae, subfamily Lamioideae," Journal of Medicinal Plants Research, vol. 2, no. 11, pp. 321-330, 2008.

[12] J. Vibes, B. Lasserre, J. Gleye, and C. Declume, "Inhibition of thromboxane A2 biosynthesis in vitro by the main components of Crataegus oxyacantha (Hawthorn) flower heads," Prostaglandins, Leukotrienes and Essential Fatty Acids, vol. 50, no. 4, pp. 173-175, 1994.

[13] K. Chen and B. Yu, "Certain progress of clinical research on Chinese integrative medicine," Chinese Medical Journal, vol. 112, no. 10, pp. 934-937, 1999.

[14] Z.-W. Zhou, H.-C. Zheng, L.-F. Zhao et al., "Effect of berberine on acetylcholine-induced atrial fibrillation in rabbit," American Journal of Translational Research, vol. 7, no. 8, pp. 1450-1457, 2015.

[15] Y.-X. Wang, Y.-M. Zheng, and X.-B. Zhou, "Inhibitory effects of berberine on ATP-sensitive K+ channels in cardiac myocytes," European Journal of Pharmacology, vol. 316, no. 2-3, pp. 307-315, 1996.

[16] S.-N. Wu, H.-S. Yu, C.-R. Jan, H.-F. Li, and C.-L. Yu, "Inhibitory effects of berberine on voltage- and calcium-activated potassium currents in human myeloma cells," Life Sciences, vol. 62, no. 25, pp. 2283-2294, 1998.

[17] B.-X. Li, B.-F. Yang, J. Zhou, C.-Q. Xu, and Y.-R. Li, "Inhibitory effects of berberine on IK1, IK, and HERG channels of cardiac myocytes," Acta Pharmacologica Sinica, vol. 22, no. 2, pp. 125131, 2001.

[18] Y. Guo, Y. Chen, Z.-R. Tan, C. D. Klaassen, and H.-H. Zhou, "Repeated administration of berberine inhibits cytochromes P450 in humans," European Journal of Clinical Pharmacology, vol. 68, no. 2, pp. 213-217, 2012.

[19] J. M. Jia, C. F. Wu, W. Liu et al., "Antiinflammatory and analgesic activities of the tissue culture of Saussurea involucrata," Biological \& Pharmaceutical Bulletin, vol. 28, no. 9, pp. 1612-1614, 2005.

[20] A. A. Damre, A. S. Damre, and M. N. Saraf, "Evaluation of sesquiterpene lactone fraction of Saussurea lappa on transudative, exudative and proliferative phases of inflammation," Phytotherapy Research, vol. 17, no. 7, pp. 722-725, 2003.

[21] A. B. Gokhale, A. S. Damre, K. R. Kulkarni, and M. N. Saraf, "Preliminary evaluation of anti-inflammatory and anti-arthritic activity of S. lappa, A. speciosa and A. aspera," Phytomedicine, vol. 9, no. 5, pp. 433-437, 2002.

[22] A. Müller, W. Linke, and W. Klaus, "Crataegus extract blocks potassium currents in guinea pig ventricular cardiac myocytes," Planta Medica, vol. 65, no. 4, pp. 335-339, 1999.

[23] M. A. Rothfuß, U. Pascht, and G. Kissling, "Effect of long-term application of Crataegus oxyacantha on ischemia and reperfusion induced arrhythmias in rats," Arzneimittel-Forschung, vol. 51, no. 1, pp. 24-28, 2001.

[24] C. J. F. Holubarsch, W. S. Colucci, T. Meinertz, W. Gaus, and M. Tendera, "The efficacy and safety of Crataegus extract WS ${ }^{\circledR} 1442$ in patients with heart failure: the SPICE trial," European Journal of Heart Failure, vol. 10, no. 12, pp. 1255-1263, 2008.

[25] M. Tauchert, "Efficacy and safety of crataegus extract WS 1442 in comparison with placebo in patients with chronic stable New 
York Heart Association class-III heart failure," American Heart Journal, vol. 143, no. 5, pp. 910-915, 2002.

[26] G. Joseph, Y. Zhao, and W. Klaus, "Pharmacologic action profile of Crataegus extract in comparison to epinephrine, amirinone, milrinone and digoxin in the isolated perfused guinea pig heart (author's translation of title)," Arzneimittelforschung, vol. 45, pp. 1261-1265, 1995.

[27] A. F. Walker, G. Marakis, A. P. Morris, and P. A. Robinson, "Promising hypotensive effect of hawthorn extract: a randomized double-blind pilot study of mild, essential hypertension," Phytotherapy Research, vol. 16, no. 1, pp. 48-54, 2002.

[28] S. R. Long, R. A. Carey, K. M. Crofoot, P. J. Proteau, and T. M. Filtz, "Effect of hawthorn (Crataegus oxycantha) crude extract and chromatographic fractions on multiple activities in a cultured cardiomyocyte assay," Phytomedicine, vol. 13, no. 9-10, pp. 643-650, 2006.

[29] R. Masteiková, J. Muselík, J. Bernatoniene et al., "Antioxidant activity of tinctures prepared from hawthorn fruits and motherwort herb," Ceska a Slovenska Farmacie, vol. 57, no. 1, pp. 35-38, 2008.

[30] J. Bernatoniene, A. Kucinskaite, R. Masteikova, Z. Kalveniene, G. Kasparaviciene, and A. Savickas, "The comparison of anti-oxidative kinetics in vitro of the fluid extract from maidenhair tree, motherwort and hawthorn," Acta Poloniae Pharmaceutica-Drug Research, vol. 66, no. 4, pp. 415-421, 2009.

[31] S. H. Kim, K. W. Kang, K. W. Kim, and N. D. Kim, "Procyanidins in crataegus extract evoke endothelium-dependent vasorelaxation in rat aorta," Life Sciences, vol. 67, no. 2, pp. 121-131, 2000.

[32] A. P. Sagare, Y. L. Lee, T. C. Lin, C. C. Chen, and H. S. Tsay, "Cytokinin-induced somatic embryogenesis and plant regeneration in Corydalis yanhusuo (Fumariaceae)-a medicinal plant," Plant Science, vol. 160, no. 1, pp. 139-147, 2000.

[33] D. J. Wang, H. Y. Mao, and M. Lei, "Rotundium in the treatment of atrial fibrillation," Zhongguo zhong xi yi jie he za zhi, vol. 13, no. 8, pp. 455-451, 1993.

[34] H. Matsuda, H. Shiomoto, S. Naruto, K. Namba, and M. Kubo, "Anti-thrombic action of methanol extract and alkaloidal components from Corydalis tuber," Planta Medica, vol. 54, no. 1, pp. 27-33, 1988.

[35] A. Frustaci, C. Chimenti, F. Bellocci, E. Morgante, M. A. Russo, and A. Maseri, "Histological substrate of atrial biopsies in patients with lone atrial fibrillation," Circulation, vol. 96, no. 4, pp. 1180-1184, 1997.

[36] D. S. G. Conway and G. Y. H. Lip, "Atrial fibrillation, the prothrombotic state, inflammation, and gender," American Journal of Cardiology, vol. 95, no. 6, p. 819, 2005.

[37] M. Ritter, K. Melichar, S. Strahler et al., "Cardiac and electrophysiological effects of primary and refined extracts from Leonurus cardiaca L. (Ph.Eur.)," Planta Medica, vol. 76, no. 6, pp. 572-582, 2010.

[38] K. Wojtyniak, M. Szymański, and I. Matławska, "Leonurus cardiaca L. (Motherwort): a review of its phytochemistry and pharmacology," Phytotherapy Research, vol. 27, no. 8, pp. 11151120, 2013.

[39] Q.-Z. Zou, R.-R. Bi, J.-M. Li et al., "Effects of motherwort on blood hyperviscosity," The American Journal of Chinese Medicine, vol. 17, no. 1-2, pp. 65-70, 1989.

[40] S. Jafari, A. Salaritabar, A. Moradi, M. Khanavi, and M. Samadi, "Antioxidant activity and total phenolic content of extracts and fractions of cultivated Leonurus cardiaca L.," Planta Medica, vol. 76, p. 376, 2010.
[41] A. Burashnikov, A. Petroski, D. Hu, H. Barajas-Martinez, and C. Antzelevitch, "Atrial-selective inhibition of sodiumchannel current by Wenxin Keli is effective in suppressing atrial fibrillation," Heart Rhythm, vol. 9, no. 1, pp. 125-131, 2012.

[42] M. Wang, Y.-B. Yu, and S.-E. Huang, "Clinical observation on effect and safety of combined use of wenxin granule and amiodarone for conversion of auricular fibrillation," Zhongguo Zhong Xi Yi Jie He Za Zhi, vol. 26, no. 5, pp. 445-448, 2006.

[43] Y. Chen, S. Nie, H. Gao et al., "The effects of Wenxin Keli on P-wave dispersion and maintenance of sinus rhythm in patients with paroxysmal atrial fibrillation: a meta-analysis of randomized controlled trials," Evidence-based Complementary and Alternative Medicine, vol. 2013, Article ID 245958, 9 pages, 2013.

[44] D. Hu, H. Barajas-Martínez, A. Burashnikov, B. K. Panama, J. M. Cordeiro, and C. Antzelevitch, "Mechanisms underlying atrial-selective block of sodium channels by Wenxin Keli: experimental and theoretical analysis," International Journal of Cardiology, vol. 207, pp. 326-334, 2016.

[45] A. Burashnikov and C. Antzelevitch, "New developments in atrial antiarrhythmic drug therapy," Nature Reviews Cardiology, vol. 7, no. 3, pp. 139-148, 2010.

[46] J. Kalifa and U. M. R. Avula, "The Chinese herb extract Wenxin Keli: atrial selectivity from the Far East," Heart Rhythm, vol. 9, no. 1, pp. 132-133, 2012.

[47] G.-R. Li, J. Feng, L. Yue, M. Carrier, and S. Nattel, "Evidence for two components of delayed rectifier $\mathrm{K}+$ current in human ventricular myocytes," Circulation Research, vol. 78, no. 4, pp. 689-696, 1996.

[48] Y. Blaauw, H. Gögelein, R. G. Tieleman, A. Van Hunnik, U. Schotten, and M. A. Allessie, "Early' class III drugs for the treatment of atrial fibrillation: efficacy and atrial selectivity of AVE0118 in remodeled atria of the goat," Circulation, vol. 110, no. 13, pp. 1717-1724, 2004.

[49] B. Pirard, J. Brendel, and S. Peukert, "The discovery of Kv1.5 blockers as a case study for the application of virtual screening approaches," Journal of Chemical Information and Modeling, vol. 45, no. 2, pp. 477-485, 2005.

[50] J. Xiao, Q. Zhao, A. H. Kebbati et al., "Wenxin Keli suppresses atrial substrate remodeling after epicardial ganglionic plexi ablation," Experimental and Clinical Cardiology, vol. 18, no. 2, pp. 153-157, 2013.

[51] W. Liu, R. Jiang, S. Ding et al., "Quality assessment of randomized controlled trials on Wenxin granule for treatment of atrial fibrillation," Zhongguo Zhongyao Zazhi, vol. 37, no. 1, pp. 109114, 2012.

[52] Y. Liu, N. Li, Z. Jia, F. Lu, and J. Pu, "Chinese medicine shensongyangxin is effective for patients with bradycardia: results of a randomized, double-blind, placebo-controlled multicenter trial," Evidence-Based Complementary and Alternative Medicine, vol. 2014, Article ID 605714, 6 pages, 2014.

[53] A.-H. Wang, J.-L. Pu, X.-Y. Qi et al., "Evaluation of shensongyangxin capsules in the treatment of paroxysmal atrial fibrillation: a randomized, double-blind and controlled multicenter trial," Zhonghua Yi Xue Za Zhi, vol. 91, no. 24, pp. 16771681, 2011.

[54] L. Shi, X.-C. Yang, X.-L. Liu, M. Zong, and Y.-L. Wu, "Effects of ShenSongYangXin on action potential and some current channels in isolated rabbit pulmonary vein cardiomyocytes," Zhonghua Yi Xue Za Zhi, vol. 89, no. 30, pp. 2142-2146, 2009.

[55] J.-Y. Ren, L.-J. Li, R.-J. Wang, H. Chen, Y.-L. Wu, and Y. Luo, "Effects of Shen-song-yang-xin capsule on ventricular 
arrhythmias in ischemia/reperfusion: experiment with rats," Zhonghua yi xue za zhi, vol. 88, no. 48, pp. 3440-3443, 2008.

[56] J.-G. Zou, J. Zhang, Z.-H. Jia, and K.-J. Cao, "Evaluation of the traditional chinese medicine shensongyangxin capsule on treating premature ventricular contractions: a randomized, double-blind, controlled multicenter trial," Chinese Medical Journal, vol. 124, no. 1, pp. 76-83, 2011.

[57] N. Li, Y.-P. Huo, K.-J. Ma, Q. Sun, and J.-J. Pu, "Effects of solution of dry powder of ShenSongYangXin capsule on sodium current and L-type calcium current in ventricular myocytes: experiment with guinea pig," National Medical Journal of China, vol. 87, no. 14, pp. 995-998, 2007.

[58] N. Li, K.-J. Ma, X.-F. Wu, Q. Sun, Y.-H. Zhang, and J.-L. Pu, "Effects of Chinese herbs on multiple ion channels in isolated ventricular myocytes," Chinese Medical Journal, vol. 120, no. 12, pp. 1068-1074, 2007.

[59] G. Chen, B. Wei, J. Wang et al., "Shensongyangxin capsules for paroxysmal atrial fibrillation: a systematic review of randomized clinical trials," PLoS ONE, vol. 11, no. 3, Article ID e0151880, 2016.

[60] M. Deng, X.-Q. Sui, S.-B. Zhu, W. Ma, Y. Xu, and Z.-M. Chen, "Clinical observation on the treatment of atrial fibrillation with amiodarone combined with Shenmai Injection," Chinese Journal of Integrative Medicine, vol. 16, no. 5, pp. 453-456, 2010.

[61] M. L. Qiu, L. Y. Li, S. C. Zang et al., Chinese Acupuncture and Moxibustion, Churchill Livingstone, Edinburgh, UK, 1993.

[62] H.-K. Xu and Y.-F. Zhang, "Comparison between therapeutic effects of acupuncture and intravenous injection of amiodarone in the treatment of paroxymal atrial fibrillation and atrial flutter," Zhongguo Zhen Jiu, vol. 27, no. 2, pp. 96-98, 2007.

[63] F. Lombardi, "Acupuncture for paroxysmal and persistent atrial fibrillation: an effective non-pharmacological tool?" World Journal of Cardiology, vol. 4, no. 3, p. 60, 2012.

[64] A. Lomuscio, S. Belletti, P. M. Battezzati, and F. Lombardi, "Efficacy of acupuncture in preventing atrial fibrillation recurrences after electrical cardioversion," Journal of Cardiovascular Electrophysiology, vol. 22, no. 3, pp. 241-247, 2011.

[65] X.-L. Zhang, M. Lou, and H.-Y. Wang, "Clinical efficacy observation on paroxysmal atrial fibrillation treated by acupuncture combined with Wenxin granule," Zhongguo Zhen Jiu, vol. 33, no. 8, pp. 686-688, 2013.

[66] S. Lee, M. S. Lee, J.-Y. Choi, S.-W. Lee, S.-Y. Jeong, and E. Ernst, "Acupuncture and heart rate variability: a systematic review," Autonomic Neuroscience: Basic and Clinical, vol. 155, no. 1-2, pp. 5-13, 2010.

[67] L. Pippa, L. Manzoli, I. Corti, G. Congedo, L. Romanazzi, and G. Parruti, "Functional capacity after traditional Chinese medicine (qi gong) training in patients with chronic atrial fibrillation: a randomized controlled trial.," Preventive cardiology, vol. 10, no. 1, pp. 22-25, 2007.

[68] G. Zheng, Q. Fang, B. Chen, H. Yi, Q. Lin, and L. Chen, "Qualitative evaluation of baduanjin (Traditional Chinese Qigong) on health promotion among an elderly community population at risk for ischemic stroke," Evidence-Based Complementary and Alternative Medicine, vol. 2015, Article ID 893215, 10 pages, 2015.

[69] X. Xiong, P. Wang, S. Li, Y. Zhang, and X. Li, "Effect of Baduanjin exercise for hypertension: a systematic review and meta-analysis of randomized controlled trials," Maturitas, vol. 80, no. 4, pp. 370-378, 2015.

[70] L. Mei, Q. Chen, L. Ge, G. Zheng, and J. Chen, "Systematic review of Chinese traditional exercise baduanjin modulating the blood lipid metabolism," Evidence-Based Complementary and Alternative Medicine, vol. 2012, Article ID 282131, 8 pages, 2012.

[71] S. Mao, X. Zhang, B. Shao et al., "Baduanjin exercise prevents post-myocardial infarction left ventricular remodeling (BEPREMIER trial): design and rationale of a pragmatic randomized controlled trial," Cardiovascular Drugs and Therapy, vol. 30, no. 3, pp. 315-322, 2016.

[72] M.-C. Hsu, T.-S. Wang, Y.-P. Liu, and C.-F. Liu, "Effects of baduanjin exercise on oxidative stress and antioxidant status and improving quality of life among middle-aged women," American Journal of Chinese Medicine, vol. 36, no. 5, pp. 815826, 2008.

[73] G. Zheng, B. Chen, Q. Fang et al., "Primary prevention for risk factors of ischemic stroke with Baduanjin exercise intervention in the community elder population: study protocol for a randomized controlled trial," Trials, vol. 15, no. 1, article no. 113, 2014.

[74] J.-S. Lai, C. Lan, M.-K. Wong, and S.-H. Teng, “Two-year trends in cardiorespiratory function among older Tai Chi Chuan practitioners and sedentary subjects," Journal of the American Geriatrics Society, vol. 43, no. 11, pp. 1222-1227, 1995.

[75] A. W. K. Chan, J. W. H. Sit, S. Y. Chair et al., "Evaluation of the effectiveness of tai chi versus brisk walking in reducing cardiovascular risk factors: protocol for a ranomized controlled trial," International Journal of Environmental Research and Public Health, vol. 13, no. 7, article 682, 2016.

[76] X.-H. Pan, A. Mahemuti, X.-H. Zhang et al., "Effect of Tai Chi exercise on blood lipid profiles: a meta-analysis of randomized controlled trials," Journal of Zhejiang University: Science B, vol. 17, no. 8, pp. 640-648, 2016.

[77] D. Jiang, W. Kong, and J. Jiang, "The role of Tai Chi in mental health management-lessons learned from clinical trials," Reviews on Recent Clinical Trials, vol. 11, no. 4, pp. 324-332, 2016.

[78] S.-M. Ng, C.-W. Wang, R. T.-H. Ho, E. T.-C. Ziea, V. C.-W. T. Wong, and C. L.-W. Chan, "Tai Chi exercise for patients with heart disease: a systematic review of controlled clinical trials," Alternative Therapies in Health and Medicine, vol. 18, no. 3, pp. 16-22, 2012.

[79] A. Burashnikov, J. M. Di Diego, A. C. Zygmunt, L. Belardinelli, and C. Antzelevitch, "Atrium-selective sodium channel block as a strategy for suppression of atrial fibrillation: differences in sodium channel inactivation between atria and ventricles and the role of ranolazine," Circulation, vol. 116, no. 13, pp. 1449-1457, 2007. 


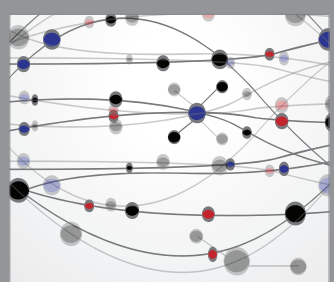

The Scientific World Journal


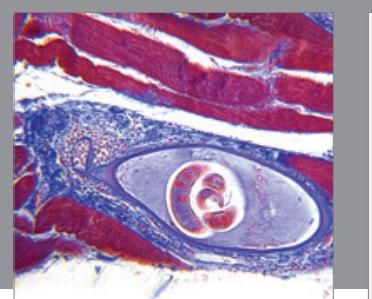

Gastroenterology Research and Practice
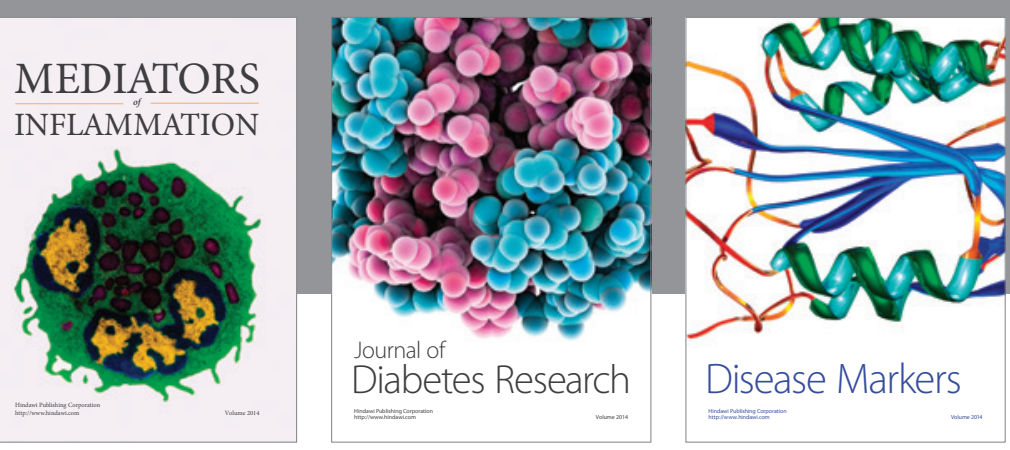

Disease Markers

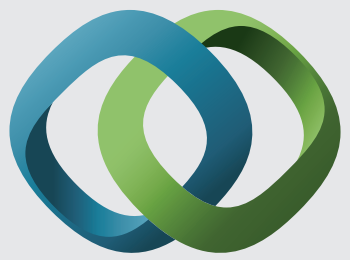

\section{Hindawi}

Submit your manuscripts at

https://www.hindawi.com
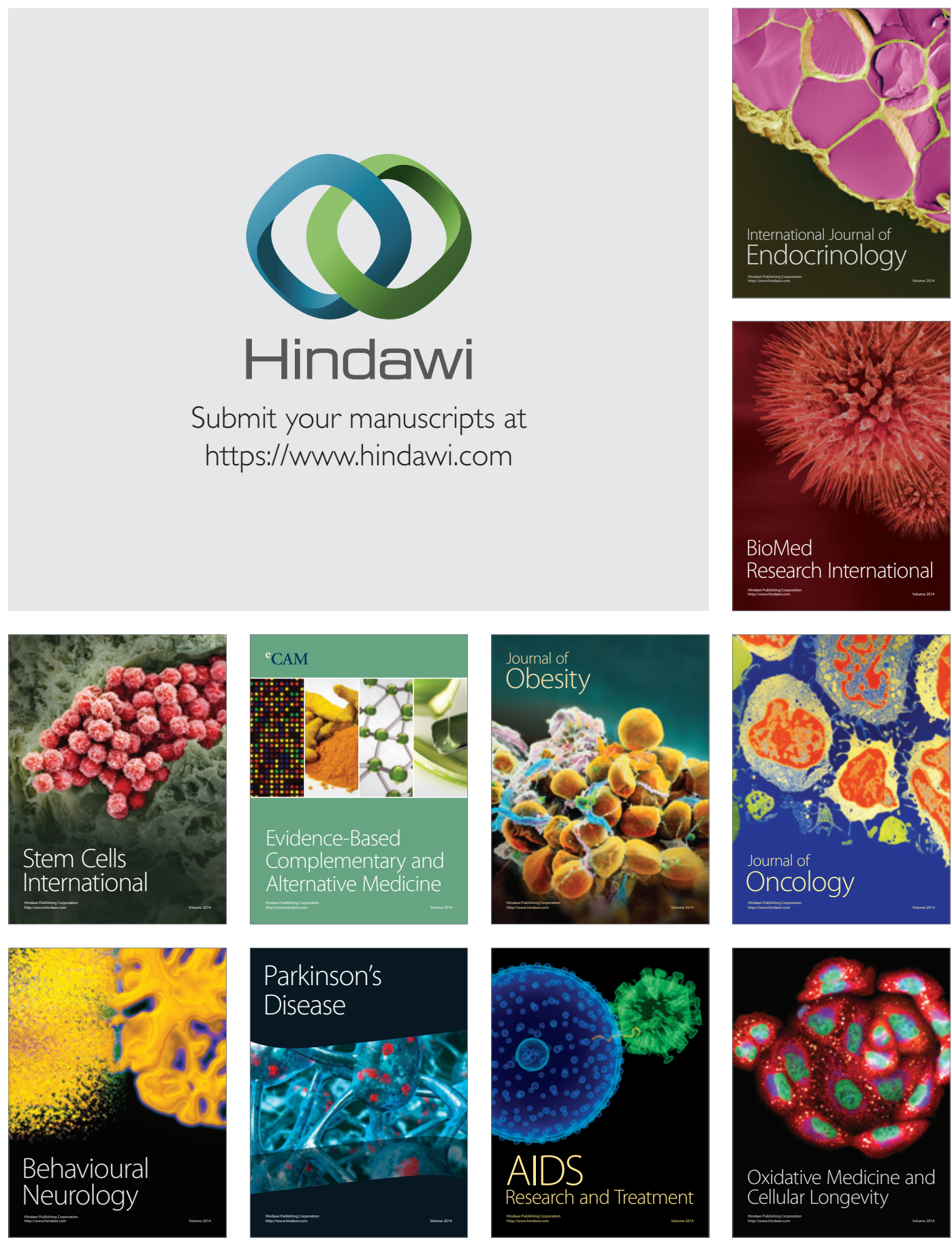